\title{
Study of fluid flow simulation and the influence of model control parameters using ENS method
}

\author{
Susithra Lakshmanan *, William J Easson \\ Institute for Materials and Processes, School of Engineering, The University of Edinburgh, United Kingdom \\ *Corresponding author E-mail: susithra@hotmail.com
}

Copyright $\odot 2015$ Susithra Lakshmanan, William J Easson. This is an open access article distributed under the Creative Commons Attribution License, which permits unrestricted use, distribution, and reproduction in any medium, provided the original work is properly cited.

\begin{abstract}
Flow in porous media is of considerable interest in many areas of engineering and science including water and pollutant flow in soils, water flow in geothermal reservoirs and flows through packed bed reactors. A three-speed Galileaninvariant LGA (GI-LGA) method has been applied to accurately describe the hydrodynamic behaviour. Influence of sub-volume size and temperature difference on viscosity is investigated in this present study. Fluid dynamics experiments such as flow between parallel plates and flow past a circular cylinder is studied; flow between two flat plates demonstrated the expected fluid dynamic behaviour and flow around a cylinder compared well with literature results in measurement of the drag coefficient.
\end{abstract}

Keywords: Explicit Numerical Simulation; Fluid Dynamic Experiments; Parameter Analysis; Single-Phase Flow.

\section{Introduction}

The phenomena of flow through porous medium occur in diverse environments in nature as well as in science and engineering applications. The study of flow in porous media plays a crucial role in the design and construction of many state-of-art, high end technological applications like biomedical (e.g. artificial organ systems), alternate energy sources (underground geothermal wells) and nuclear technology (reactors and treatment of waste nuclear effluents). Many different models of fluid transport in porous media have been described in the literature [1-2]. Lattice-gas automata based explicit numerical simulation (ENS) tool has been used to simulate many different flow situations [3-6] and this technique solve microscopic equations for moving and interacting fluid particles on a discrete lattice. A range of applications where higher speed flows through porous system and transitions between the different flow regimes and identifying the heat and mass transfer characteristics are important in the above mentioned industrial process. Hence extension of the ENS approach to higher speed flows in porous media is important.

LGA simulation techniques for fluid transport, offer several advantages; the algorithm is completely stable, a feature derived from the absence of round-off, costly floating-point calculations may be replaced by fewer boolean and/or table-lookup operations. However they are restricted to relatively slow flows due to non-Galilean invariance. The Galilean invariance factor present in the hydrodynamic equations is an artefact introduced by the limited number of particle momentums and the discrete lattice used. There is a class of LGA models known as multi-speed LGA which use multiple lattice particle speeds [7-9] and remove this artefact of the lattice from the hydrodynamic equations and recover continuum hydrodynamics exactly. The details of the implementation have not been published in the open literature including the influence of the various control parameters of the method.

The present study aimed at elucidating the influence of the various control parameters of the method and applied to fluid dynamic experiments to demonstrate that the model produces correct results whilst recovering Galilean invariance. This study not only essential for higher speed flows, but also constitutes the basis for determining non-Darcy transport properties and regime transitions for a flow through porous medium is of particular interest. 


\section{Materials and methods}

\subsection{Overview of LGA based ENS model}

Frisch et al [4] showed that with suitable collision rules and other moderating conditions such as density in place, the collective motion of the particles should yield behaviour similar to that predicted by the incompressible Navier-Stokes equations with the presence of density dependent factor. The complete derivation has been discussed by various authors [10-11]. To summarise, they used the principles of kinetic theory to derive a Boltzmann-like equation for the LGA particle dynamics and solved it using a Chapman-Enskog approach to yield the following hydrodynamic equations (equations 1.1, 1.2) and equation of state (equation 1.3) for the LGA fluid.

$\frac{\partial \rho}{\partial t}+\nabla \cdot(\rho u)=0$

$\frac{\partial(\rho u)}{\partial t}+\nabla \cdot[\rho g(\rho) u u]=-\nabla p+\gamma(\rho) \nabla^{2}(\rho u)$

$p=c_{S}^{2} \rho\left(1-g(\rho) \mathrm{u}^{2}\right)$

$\rho$ And $u$ are the macroscopic density and velocity and $c_{s}$ and $v(\rho)$ are the speed of sound and kinematic viscosity respectively. The momentum equation differs significantly from that of the Navier-Stokes equation due to the presence of the non-unitary $g(\rho)$, Galilean invariance factor in the momentum convection term. The Galilean invariance factor present in the hydrodynamic equations (equation 1.3) is an artefact introduced by the limited number of particle momentums and the discrete lattice used. If this Galilean invariance factor is not 1 (i.e. the lattice fluid is non-Galilean invariant), this manifests as the vorticity advecting with a different velocity to the bulk movement of the fluid [10]. A variety of methods have been proposed to remove the various non-hydrodynamic artefacts from LGA [9-12]. Perhaps the most advanced is Teixeira model which recovers Galilean invariance in three-dimensions by allowing particles to take on one of a small number (e.g. three) speeds and then controlling the density of each over small sub-volumes (e.g. $5 \times 5 \times 5$ lattice units). Few papers that have discussed this issue [8-9] and reported only the results with very few details. This section, therefore, provides a comprehensive summary of our implementation of the Teixeira method.

\subsection{Implementation}

FCHC model resides on an ordinary 3D cubic lattice with the velocity structure is maintained with unit periodicity in the fourth direction and the lattice structure is shown in figure 1. Total of 54 particles in which 24 belong to energy1, other 24 belong to energy 2 and 6 energy0 particles were used in this model.

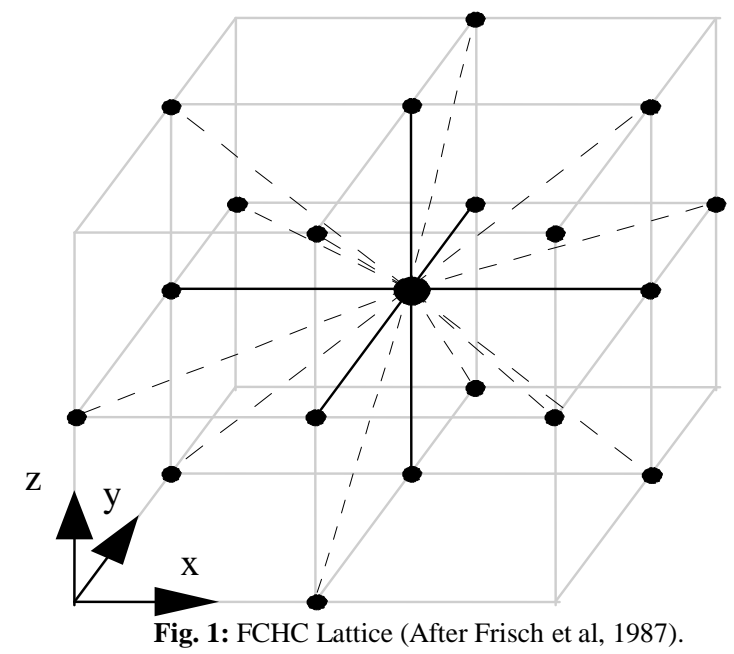

The structure of the underlying 4D FCHC lattice allows these particles of different speeds to exist independently on sufficiently symmetric sub lattices while also allowing particles of different speeds to interact via energy exchange collisions. These energy exchange collisions force the lattice artefacts to have the continuum values necessary for correct hydrodynamic behaviour. 


\subsubsection{Initialization of the lattice}

The initialization part sets up the geometry on the entire lattice according to a user-defined function. The equilibrium distribution is done by seeding the particles with the probabilities equal to the equilibrium Fermi-Dirac distribution function. This ensures not only the conserved quantities of mass, momentum and energy, but also allows the Galilean invariant factor ' $\mathrm{g}$ ' to be set at 1 , i.e. $\mathrm{g}=1$ as well. The relation for $\mathrm{g}$ is in terms of moments of the equilibrium distribution function as

$$
N_{j}=\frac{y r_{j}}{y r_{j}+z^{j}}
$$

Where $\mathrm{y}$ and $\mathrm{z}$ are quantities related to density and temperature and $\mathrm{r}$ is the rate coefficient. The equilibrium distributions for this model could be calculated as following:

$$
\begin{aligned}
& N_{0}=\frac{y r}{y r+1} \\
& N_{1}=\frac{y}{y+z} \\
& N_{2}=\frac{y}{y+z^{2}}
\end{aligned}
$$

The density and energy relations for the three speed model are

$$
\begin{aligned}
& f=\frac{1}{4} N_{0}+N_{1}+N_{2} \\
& U_{p}=24\left(N_{1}+2 N_{2}\right)
\end{aligned}
$$

Using the above equations, the Galilean invariance condition can be written in the following equation for this model

$g=\frac{2}{3}\left[\frac{N_{1}\left(1-N_{1}\right)\left(1-2 N_{1}\right)+4 N_{2}\left(1-N_{2}\right)\left(1-2 N_{2}\right)}{\left(N_{1}\left(1-N_{1}\right)+2 N_{2}\left(1-N_{2}\right)\right)^{2}}\right] f=1$

One way of assuring the rate coefficient $r$ remains at the appropriate value is to create a feedback system that can sense the local density and temperature related variable $(\mathrm{z})$ and then alter the collisions accordingly.

\subsubsection{Addition of solid surfaces}

Certain lattice sites are labelled as a solid surface in the model. To assign a node as a solid state an additional bit is used to store the corresponding node information. It denotes whether the node is part of a solid surface or not. Depending on the level of shear stresses experienced between the LGA fluid and the solid surface, a slip or no-slip boundary condition is assigned to the solid surface [13]. This is done by using spectacular reflection or bounce-back collision rules respectively implemented at solid nodes or a mixture of the two [14-17]. Bounce-back reflections are used in our implementation. This is shown in figure.2.

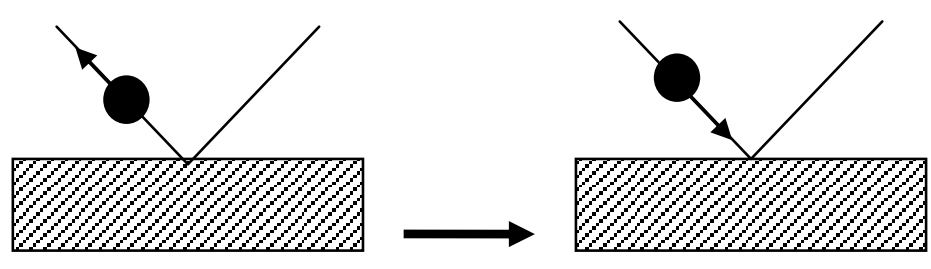

Fig. 2: Bounce-back collision at a solid node.

\subsubsection{Periodic boundaries}

Simulation domains have been coded with default periodic boundary conditions, i.e. any particles leaving one side of the simulation domain will re-enter on the opposite side. To encode this into the current models, an additional plane of lattice nodes outside of the simulation domain is included on each side of the lattice. These nodes contain the same information as the plane of nodes on the opposite side of the lattice. This information is copied across once in each time-step before the propagation stage, to allow the propagation of particles to nodes on the edge of the lattice. 


\subsubsection{Applying force}

Body force is applied in LGA by introducing an additional "forcing" stage to the automaton's updating algorithm. A force may be thought of as a rate of change of momentum. Hence a force is applied by changing the momentum states of a set number of lattice particles at each time state. This will require a particle be present in a momentum state from which it may be moved and a corresponding "hole" to move it into. If these conditions are met, the particle is moved into its new momentum state. Another node is then chosen and this operation repeated until the required force has been applied.

\subsubsection{Collision mechanism}

Each collision is a binary collision occurring between two sets of paired particles. The collisions are classified into two groups: self-collisions and energy exchange collisions. In the self-collisions; collisions between energy 1 particles and collisions between energy 2 particles are considered. Energy exchange collisions occur when two energy 1 particles collide to form an energy 2 particle and an energy0 particle. The inclusion of energy exchange collisions adds an additional degree of freedom to the system in order to simulate the true fluid behaviour. The energy exchange collision happens in forward direction from the energy 1 particles and a backward direction from the energy 2 and energy 0 particles. These collisions rates are dependent on the temperature of the system. A feedback system is created to sense the local density and temperature and then alter the collisions accordingly. The Galilean invariance factor ' $\mathrm{g}$ ' is directly affected by the rate of energy exchange collisions. By regulating the number of energy transfer collisions the model is forced to exhibit Galilean invariance.

\subsubsection{Macroscopic property}

The final step in the entire algorithm is the calculation of macroscopic properties of the flow for all 54 particles. At any node the local value of fluid density, momentum or any other macroscopic flow variable is calculated by taking appropriate bits of the microscopic distribution as below.

$$
\begin{aligned}
& \rho(x)=\sum_{i=1}^{54} n i(x) \\
& \rho u(x)=\sum_{i=1}^{54} c_{i} n i(x)
\end{aligned}
$$

This expression calculates the local values of density and momentum respectively accumulated over a sub-volume. It is divided by the size of sub-volume to get an average quantity per lattice node.

\section{Results and discussion}

\subsection{Effect of model control parameters on performance}

To determine the equilibrium temperature of the system, optimization studies has been carried out. The entire lattice is divided into small sub-volumes and the temperature is defined with an upper and lower temperature range. The effects of sub-volume size and temperature difference $(\Delta \mathrm{T})$ were examined and the results are discussed in this section. Both factors are very important in deciding the outcome of the process. Poiseuille flow experiments were used in this study.

\subsubsection{Effect of sub-volume size}

It is important to predict the sub-volume size which is independent of viscosity for further simulations. The resolution of the lattice is selected based on the viscosity of the flow. Sub-volumes are grouped as micro blocks in to various sizes. The following for sub-volume sizes, $(4,4,4),(6,4,4),(12,4,4)$ and $(29,4,4)$, were used in the simulations. The nodes in the sub-volume is defined as $N_{i}(n)$, where $\mathrm{n}$ represents the relative position of the lattice node within the sub-volume and $n \in\{0,1,2, \ldots . .63\}$ for the sub-volume of dimensions $\mathrm{X}=4, \mathrm{Y}=4, \mathrm{Z}=4$ as grouped as $(4,4,4)$. Simulations were carried out by varying the sub-volume sizes for constant temperature difference $(\Delta \mathrm{T})$ range. Viscosity fluctuation as a function of sub-volume size (in terms of total number of nodes) is plotted in figure 3. 


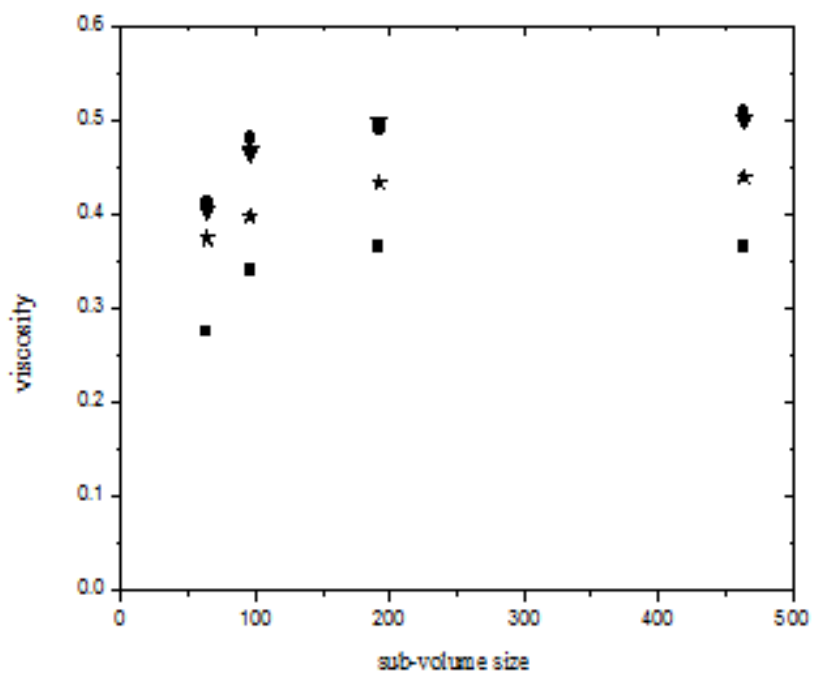

Fig. 3: Viscosity fluctuation as a function of sub-volume size: $\| \Delta \mathrm{T} 0.015, \square \Delta \mathrm{T} 0.0165, \boldsymbol{\nabla} \Delta \mathrm{T} 0.0175, \bullet \Delta \mathrm{T} 0.0185$.

Keeping the $\Delta \mathrm{T}$ at $0.015,0.0165,0.0175$ and 0.0185 , various sub-volume sizes were analysed. The results showed a trend of initial increase in viscosity before it become constant with increasing sub-volume size. Increasing the subvolume size beyond $(12,4$, and 4) did not influence the viscosity. This is probably because the system gets saturated with regard to the collisions rules as it is pre-defined in the model.

\subsubsection{Effect of temperature difference $(\Delta T)$}

Further simulations were carried out for various temperature differences while keeping the sub-volume size constant to see the viscosity changes. This will also enable us to verify the accuracy of previous simulation results. For each of the sub-volume size $(4,4,4),(6,4,4),(12,4,4),(29,4,4)$ simulations were carried out with different $\Delta \mathrm{T}$ values. Viscosity fluctuation as a function of temperature difference $(\Delta \mathrm{T})$ range is plotted in figure 6 . The change in viscosity pattern was similar with initial increase before it become constant at 0.0175 . As before the predetermined model parameters make the system saturated with maximum number of collisions. The viscosity constant regions in the above studies were selected for the following fluid dynamic experiments.

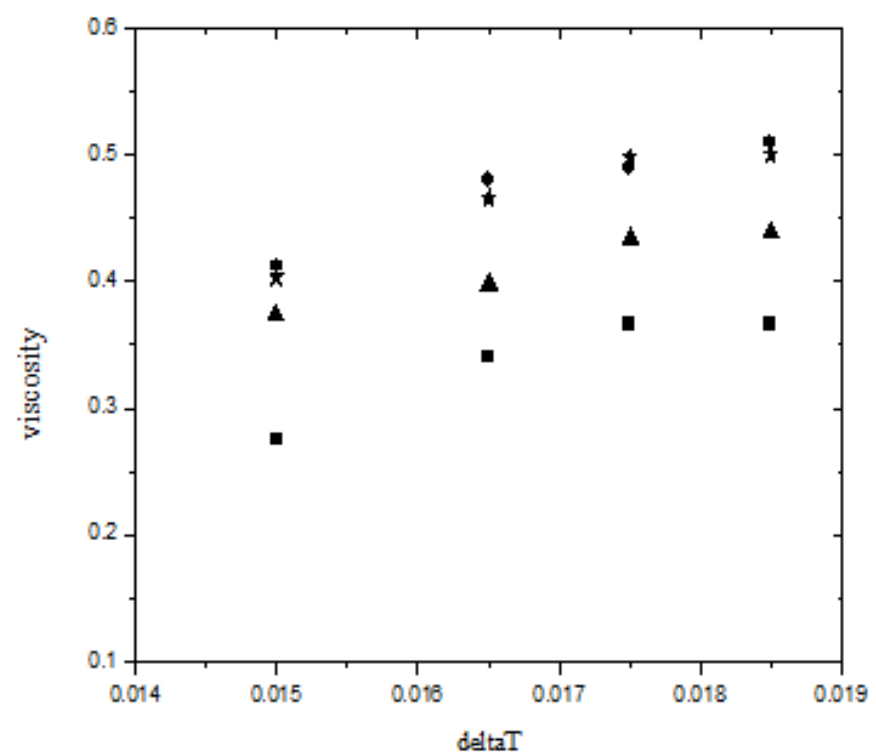

Fig. 4: Viscosity fluctuation as a function of temperature difference $(\Delta \mathrm{T})$ : $\boldsymbol{\bullet}$ Sub-volume Size 4, 4, 4, $\boldsymbol{\Delta}$ Sub-volume Size 6, 4, 4, $\square$ Sub-volume Size 12, 4, 4, $\bullet$ Sub-volume Size 29, 4, 4

\subsection{Validation of Galilean invariance artefact removal}

Relaxation of a shear wave with transverse velocity in an open system [18] is examined for the removal of Galilean invariance artefact, $\mathrm{g}$. The lattice is initialized with particular density and temperature and an initial velocity field is given by 
$u_{0}(x, t=0)=u_{T} e_{y}+u_{L} \sin (k y) e_{x}$

Where $k$ is the wave number of the perturbation. Having a non-zero streaming speed in the direction of the perturbation will allow $g$ to be measured directly from the evolution of the perturbation. The evolution of this perturbation in time is given by the lattice Navier-Stokes equation;

$\frac{\partial u}{\partial t}+g u . \nabla u=\gamma \nabla^{2} u$

Substituting equation 1.11 for $\mathrm{u}$ in the above equation, it can be calculated analytically. The following solution can be derived.

$u(x, t)=u_{T} e_{y}+u_{L} e^{-i k u_{T} g t-\gamma k^{2} t} \sin (k y) e_{x}$

Simulations were run on a $64 \times 64 \times 64$ lattice. Validation runs were done with $u_{T}=u_{L}=0.1$ and a wave number of k $=2 \pi / 64$ which corresponds to one wavelength of the perturbation exactly fitting into the lattice volume. All macroscopic flow velocities are in units of number of lattice nodes travelled per time step. The component of momentum in the x-direction was measured at every time step and this signal was then Fourier transformed and the component with wave number $\mathrm{k}$ was extracted, all other Fourier components being noise. Phase and modulus of the remainder was calculated. Representative plots of the phase and modulus are shown in figure 5 and 6.

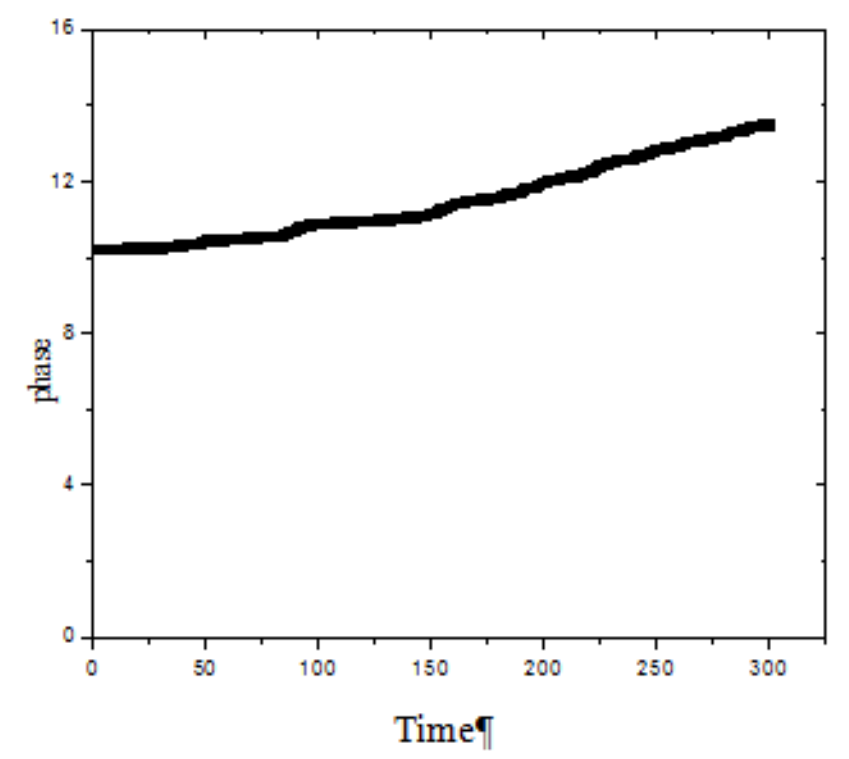

Fig. 5: Phase of the shear perturbation as a function of time.

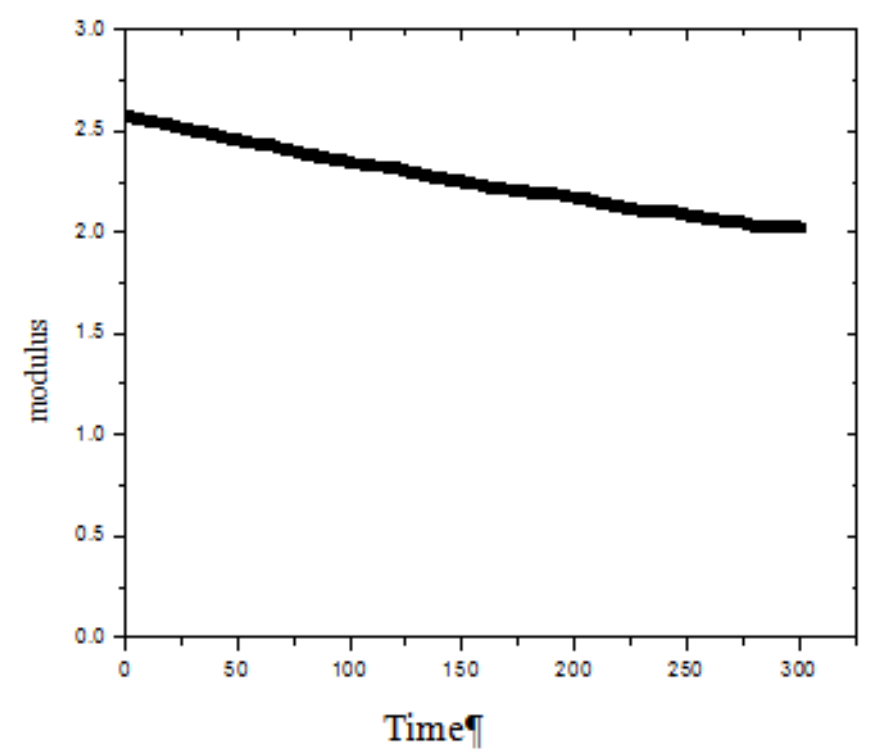

Fig. 6: Modulus of the shear perturbation as a function of time. 
The slope of the phase evolution is equal to $g k u_{T}$ in figure 4.6 and the slope of the modulus as function of time is equal to $\gamma \mathrm{k}^{2}$ in figure 6. Since $k$ and $u_{T}$ are input parameters it was possible to calculate $g$ and $\gamma$ from these slopes. The slope in figure 5 yield $g=1.028 \pm 0.02$. It is evident from this result that this model achieves macroscopic Galilean invariance.

\subsection{Fluid dynamic experiments}

We examined some of the fluid dynamics experiments such as flow between parallel plates and flow past a circular cylinder. The results are compared various flow properties with available experimental results.

\subsubsection{Poiseuille flow}

The lattice is initialized with particles of density $\rho$ and temperature related variable z. GI-LGA algorithm is applied to simulate the flow in a regular FCHC lattice. With the $\mathrm{x}$-direction being aligned to the length of the channel, the NavierStokes equation for this flow simplifies to

$\frac{\partial}{\partial t} u_{x}=-\frac{1}{\rho} \frac{\partial}{\partial x} p+v \frac{\partial^{2}}{\partial y^{2}} u_{x}$

In a steady state condition, the flow is zero at the walls and reaches a maximum in the center. The solution is

$u_{x}(y)=u_{0}\left(1-\frac{y^{2}}{a^{2}}\right)$

Where $a$ is the half-height of the channel centered around $y=0$. At equilibrium the velocity should attain a parabolic profile throughout the channel. From this result, a viscosity measurement can be obtained. The simulation was started with the fluid at rest. Once the equilibrium was reached the data was accumulated for another 2000 time steps to assure the clean profile and measurements. A sample velocity distribution is shown in figure 7 . In the figure we show both the theoretical and simulation result and the channel flow gives the expected parabolic profile to a good degree of accuracy. This is further strong evidence that the algorithm exhibits correct hydrodynamic behaviour as expected.

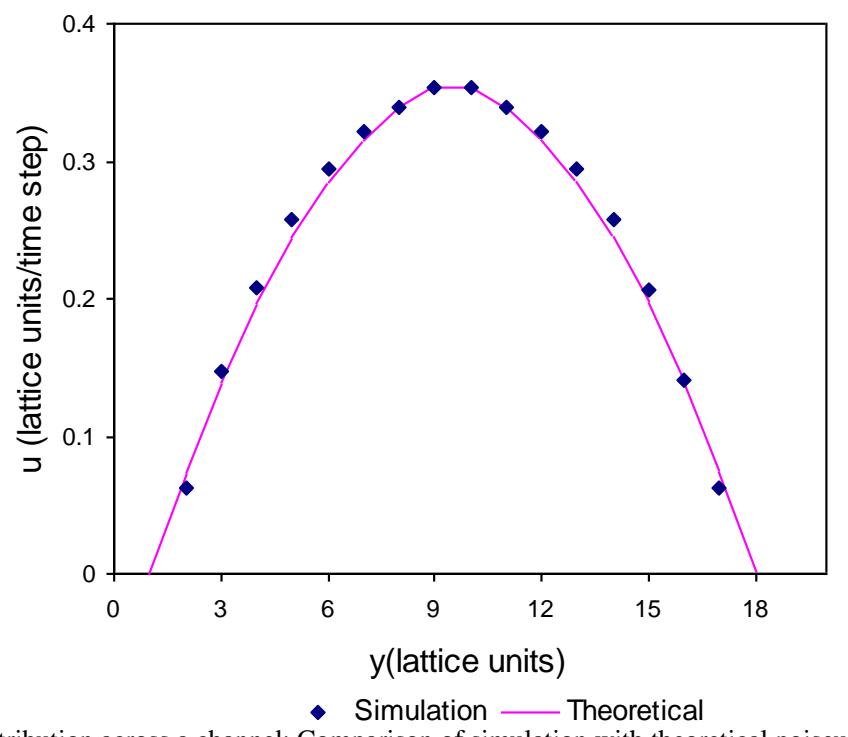

Fig. 7: Flow distribution across a channel: Comparison of simulation with theoretical poiseuille flow profile.

\subsubsection{Flow past a circular cylinder}

Cylinder was created in the LGA coding by assigning the nodes corresponding to the cylinder dimensions as a solid boundary. By setting the initial density and temperatures as constants, flow velocity is varied to obtain different Reynolds numbers.

Reynolds number is one of the important concepts in the fluid dynamics. It is a dimensionless number and it is defined as the ratio of inertial to viscous force. It quantifies the contribution of these forces in particular flow regime. The Reynolds number can be calculated by the following

$\operatorname{Re}=\frac{u d}{v}$ 
Where $\mathrm{d}$ is the diameter of the cylinder in lattice unit, $\mathrm{u}$ is the fluid velocity and $v$ is the kinematic viscosity of the fluid. The lattice sizes were chosen from those lattice sizes reported in the literature to facilitate the comparison of the results. Once the steady state had been reached, the drag on the cylinder was calculated to determine the drag coefficient

$$
C_{d}=\frac{F_{c y l}}{A_{C S} \cdot \frac{1}{2} \rho u^{2}}
$$

Where $F_{c y l}$ the total is force on the cylinder and $A_{C S}$ is the frontal cross-section of the cylinder which is equal to twice the radius multiplied by the cylinder length. The force deposited to the wall by one particle is the amount of momentum imparted to the wall by the particle over the duration of the collision. The drag coefficient as a function of the Reynolds number is plotted in figure 8 .

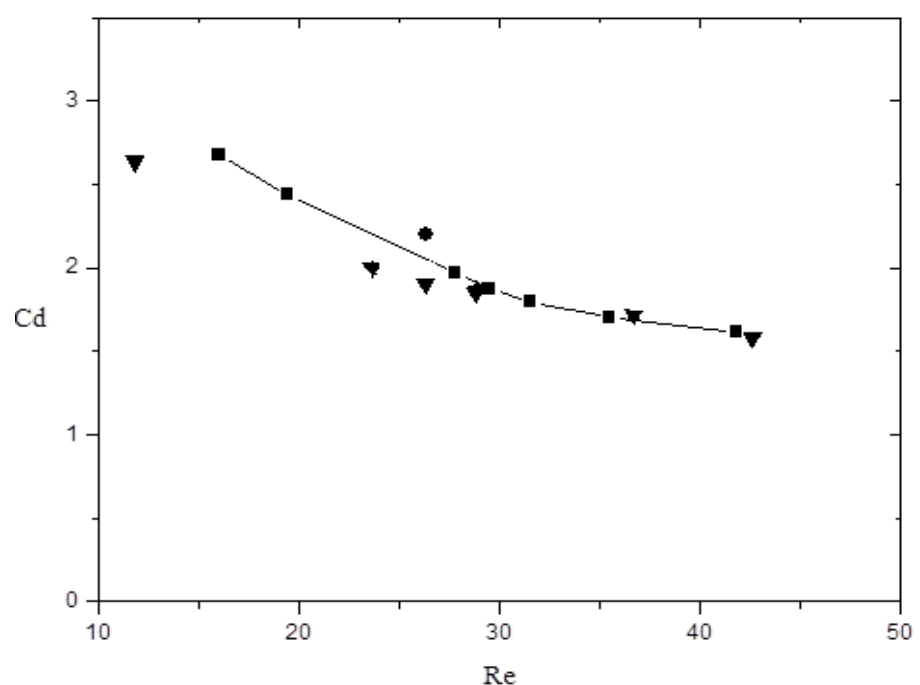

Fig. 8: $C_{d}$ Vs. Re. Simulation Results (•), Experimental Results ( $\left.\mathbf{\nabla}\right)$, CFD Result $(\bullet)$. The line connects the simulation results for clarity.

Our results are comparable with the experimental results of Tritton and CFD results of Karniadakis [19-20]. Quantitative agreement between our data and the literature results is satisfactory for $\operatorname{Re}>25$. There is a small discrepancy between our results and the literature results for low Reynolds number. One possibility for the disagreement at lower Re could be the influence of surface roughness for smaller radius cylinder and this issue concerns the degree to which we can delineate a continuously curving cylinder in a discrete lattice.

\section{Conclusions}

A three-speed GI-LGA model has been successfully implemented to accurately describe the hydrodynamic behaviour by removing the Galilean invariance artefact $\mathrm{g}$. It is validated through a shear wave experiment. The influence of subvolume size and temperature difference on viscosity is studied. An application of this model to the flow between two flat plates demonstrated the expected fluid dynamic behaviour. Flow around a cylinder compared well with literature results in measurement of the drag coefficient. This study illustrates the suitability of this simulation model could be used to study the fluid dynamics of non-Darcy flow through porous medium, high end technological applications include packed bed and membrane reactors.

\section{Acknowledgements}

We acknowledge the EPSRC PhD studentship and The University of Edinburgh Research Scholarship for SL.

\section{References}

[1] Dullien, F.A.L., 'Porous Media - Fluid Transport and Pore Structure', Academic Press Inc., London-UK, 1992.

[2] Sahimi, M., Flow and Transport in Porous Media and Fractured Rock: From Classical Methods to Modern Approaches, John Wiley, Hoboken, N. J, 1995.

[3] Wolfram, S.,'Lattice Gas Methods for Partial Differential Equations', Journal of Statistical. Physics. Vol 45 : $471-526$, 1986. http://dx.doi.org/10.1007/BF01021083.

[4] Frisch, U., d'Humières, D., Hasslacher, B., Lallemand, P., Pomeau, Y., and Rivet, J-P., Lattice Gas hydrodynamics in two and three dimensions, Complex Systems, Vol 1: 649 -707, 1987. 
[5] Chen,S., Dawson.S.P., Doolen,G.D., Janecky,D.R., Lawniczak,A., Lattice methods and their applications to reacting systems, Computers \& Chemical Engineering, Vol 19:7617-646, 1995. http://dx.doi.org/10.1016/0098-1354(94)00072-7.

[6] Biggs, M.J. and Humby, S. J.,Lattice-gas automata methods for engineering, Transactions of the IChemE, 76:162-174, 1998. http://dx.doi.org/10.1205/026387698524730.

[7] Molvig.K, Donis, P., Miller, R., Myczkowski,J., and Vichniac, G., Multi-Species Lattice-Gas Automata for Realistic Fluid Dynamics Cellular Automata and the Modeling of Complex Physical Systems, Springer proceedings in Physics, Vol 46:206-231, 1990 .

[8] Mujica .R, Lattice gas wind tunnel, MSc thesis, MIT, 1991.

[9] Teixeira, C.M., Continuum limit of Lattice gas fluid dynamics, PhD Thesis, 1992.

[10] Rothman, D. H., and Zaleski, S., Lattice-gas models of phase separation: Interfaces, phase transitions, and multiphase flow, Review of Modern Physics, Vol 66:1417-1479, 1994. http://dx.doi.org/10.1103/RevModPhys.66.1417.

[11] d'Humières, D and Lallemand, P., Numerical simulations of hydrodynamics with lattice gas automata in two dimensions, Complex Systems Vol 1:599-632,1987.

[12] D’Humières, D., Lallemand, P., and Frisch, U., Lattice gas models for 3-D hydrodynamics, Europhys. Lett. Vol 2:291-297, 1986. http://dx.doi.org/10.1209/0295-5075/2/4/006.

[13] Thompson,P., and Robbins,M.,To Slip or Not to Slip?, Physics World, pp 35, 1990.

[14] Lavallée, P., Boon, J.P., and Noullez, A., in Discrete Kinetic Theory, Lattice Gas Dynamics and Foundations of Hydrodynamics, ed. R. Monaco, World Scientific, pp 206-214, 1989.

[15] Lavallée, P., Boon, J.P., and Noullez, A., Boundaries in Lattice Gas Flows, Physica D., Vol.47:233-240, 1991. http://dx.doi.org/10.1016/01672789(91)90294-J.

[16] Hayot, F., and Lakshmi, M.R., Cylinder wake in lattice gas hydrodynamics, Physica D., Vol 40: 415-420,1989. http://dx.doi.org/10.1016/0167-2789(89)90053-5.

[17] Gao.Y and Sharma, M.M., LGA model for fluid-flow in heterogeneous porous-media. Transp. Porous Media, Vol 17:1-17, 1994. http://dx.doi.org/10.1007/BF00624047.

[18] Salem.J, and Wolfram, S., Theory and Applications of Cellular Automata, S.Wolfram ed., 382, World Scientific, 1986.

[19] Tritton, D. J., Experiments on the flow past a circular cylinder at low Reynolds numbers, Journal of Fluid Mechanics Vol 6:547-567, 1959. http://dx.doi.org/10.1017/S0022112059000829.

[20] Karniadakis.G, Numerical Simulations of forced convection heat transfer from a cylinder in crossflow, Int.J.Heat.Mass.Transfer Vol 31:107118, 1988. http://dx.doi.org/10.1016/0017-9310(88)90227-X. 